\title{
Interaction of Mesenchymal Stem Cells with Poly(lactide-co- glycolide)/Hydroxyapatite Composite Scaffolds
}

\author{
Yuliya Nashchekina ${ }^{1}$, Vladimir Botvin ${ }^{2}$, Sergey Nadezhdin ${ }^{3}$, Svetlana Alexandrova ${ }^{1}$, \\ Lubov Pokrovskaya ${ }^{2}$, Miralda Blinova ${ }^{1}$, Natalia Mikhailova ${ }^{1}$ \\ ${ }^{1}$ Institute of Cytology of the Russian Academy of Science \\ Tikhoretsky ave. 4, 194064 Saint-Petersburg, Russia \\ ulychka@mail.ru; alekssvet2205@gmail.com; mira.blinova@mail.ru; natmik@mail.ru \\ ${ }^{2}$ National Research Tomsk State University \\ Lenin ave., 36, 634050, Tomsk, Russia \\ botvinilo1991@gmail.com; pokrovskayal@ect-center.com \\ ${ }^{3}$ Belgorod State University \\ Pobedi str., 85, 308015, Belgorod, Russia \\ sergey_nadezhdin@yahoo.com
}

\begin{abstract}
The aim of this study was to determine the cytocompatibility of poly(lactide-co-glycolode)/hydroxyapatite composite scaffolds. The obtained results indicated that the presence of biphasic HA/ $\beta$-TCP ceramic in poly(lactide-coglycolode)/hydroxyapatite composite scaffolds improved the adhesion and proliferation of the human fetal mesenchymal stem cells.
\end{abstract}

Keywords: Hydroxyapatite, Tri-calcium phosphate, Mesenchymal stem cell.

\section{Introduction}

The modern research effort is prepared of effectual scaffold-based strategies for tissue engineering1. The main objective in the ongoing studies is to improve the biocompatibility of the polymer scaffolds in order to control cellular behavior and tissue regeneration 2. Recently, biodegradable polymeric biomaterials get much interest in terms of formation of composite scaffolds that could serve as systems for cell growth and transplantation s6-8. Currently, the biocompatibility of poly (lactide-co-glycolode) (PLGA) has become a subject of intense investigation, due to broad range of its properties.

The design of bioactive composite scaffolds with required structural, mechanical and biological properties is a central topic of bone tissue engineering. Hydroxyapatite (HA) and tri-calcium phosphate (TCP) are widely used for bone tissue regeneration. Major limitations of these ceramic materials are brittleness, low mechanical stability and poor osteoconductivity. Moreover, biodegradation and bioresorbability of HA are quite low, as this material remains in the body for a long time after implantation. Our aim was to analyze the interaction between mesenchymal stem cells and the obtained HA/PLGA composite scaffolds.

\section{Materials and Methods}

Poly(lactide-co-glycolide) (PLGA) (L/G ratio 80:25) was syntheses by ring opening polymerization. The biphasic HA/ $\beta$-TCP ceramic was synthesis by using microwave radiation and hydrothermal conditions. Composite scaffolds with different material compositions were produced using an electrospinning method. The biphasic HA/ $\beta$-TCP ceramic and composite scaffolds were physically and chemically investigated considering mechanical, wettability, scanning electron microscopy and thermal gravimetric tests. Effect of the composite scaffolds on adhesion, proliferation and differentiation of human fetal mesenchymal stem cells (FetMSC) was investigated. Fluorescence microscopy of FetMSCs on scaffolds after staining Calcium Green-1 AM (Thermo Fisher Scientific, USA).

\section{Results and discussion}

The obtained biphasic HA/ $\beta$-TCP ceramic had highly dispersed bioresorbable powder with two-phase calcium phosphate and elemental composition $\mathrm{Ca} / \mathrm{P} \sim 1.51$. The surface morphology of analyzed biphasic HA- $\beta$-TCP ceramic is 
close to natural bone tissue. X-ray phase analysis showed the presence of the phase $\beta$-TCP $(\geq 90 \% \beta$ - TCP), with a higher degree of bioresorption compared with HA. Specific surface area was 2-10 м2/g.
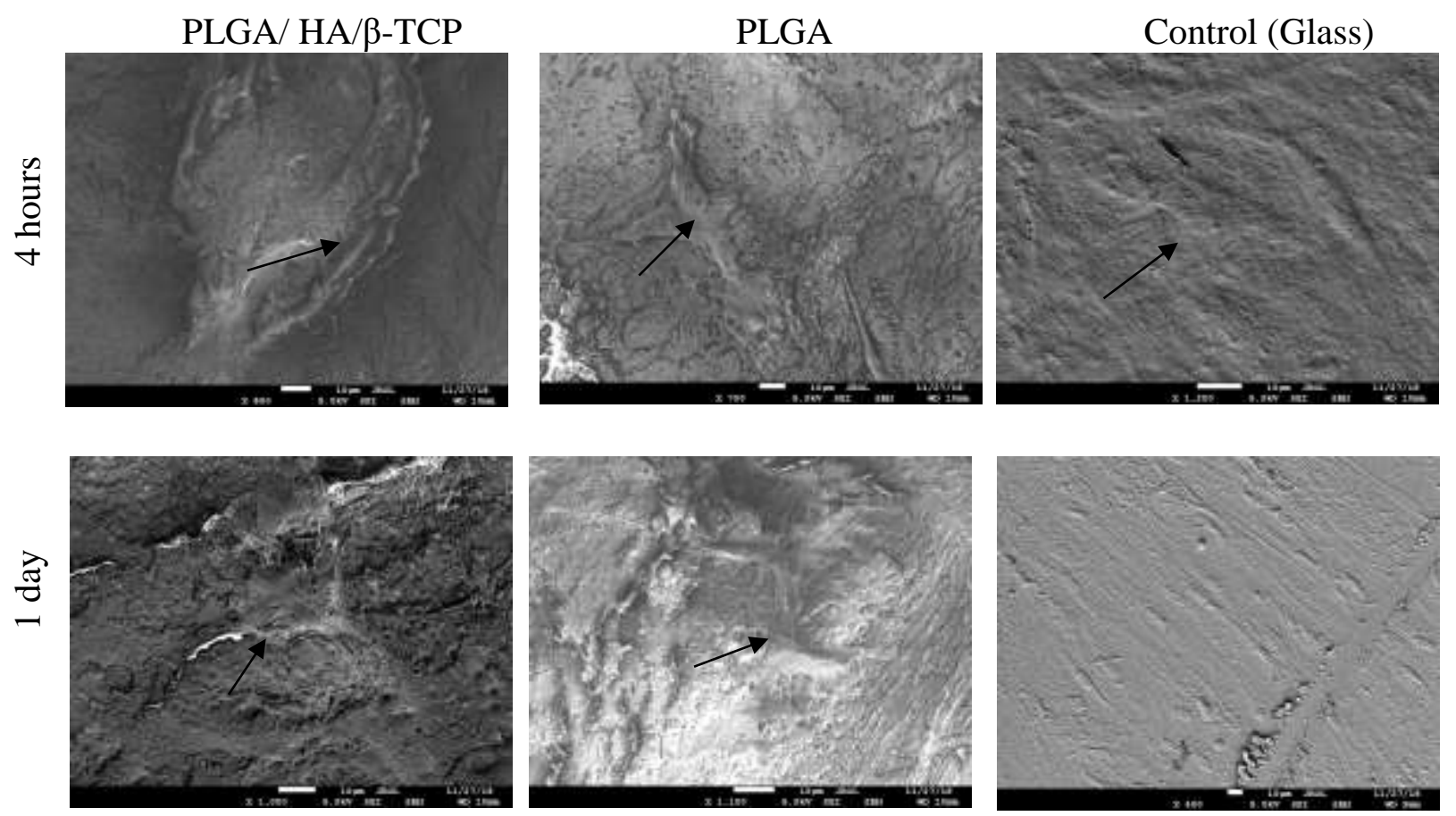

Fig. 1: SEM images of FetMSCs on scaffolds.

Results demonstrated that scaffolds containing biphasic HA/ $\beta$-TCP ceramic present better biological properties and TCP scaffolds present improved mechanical properties compare to poly(L,L-lactide-co-glycolide) scaffolds without HA$\beta$-TCP. Results of scanning electron microscopy (SEM) demonstrated that after 1 day cultivation cells are well spread (Fig. 1)

Cell proliferation after 5 days of cultivation on composite scaffolds in the presence of HA/ $\beta$-TCP is significantly higher compared to composite scaffolds without HA/ $\beta$-TCP (Fig. 2).
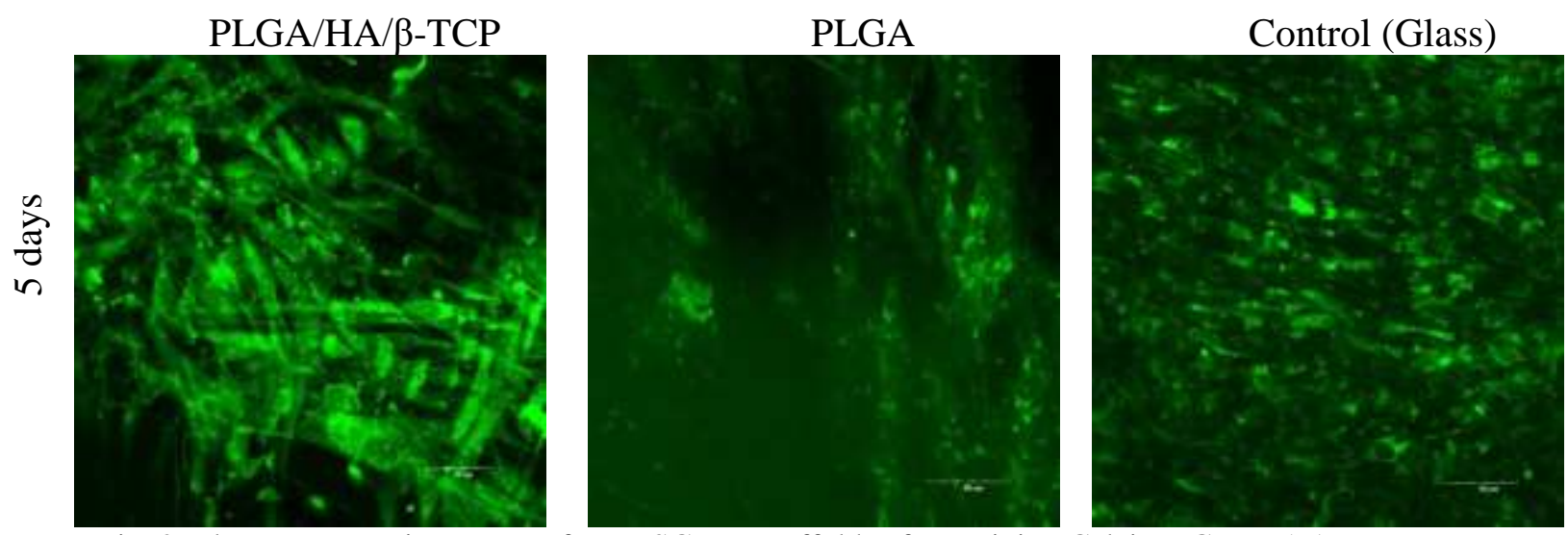

Fig. 2: Fluorescence microscopy of FetMSCs on scaffolds after staining Calcium Green-1 AM.

The MTT method has demonstrated that Toxic effect of HA- $\beta$-TCP in composition of HA/ $\beta$-TCP/poly(lactide-coglycolode) composite scaffolds was not detected (Fig. 3). 


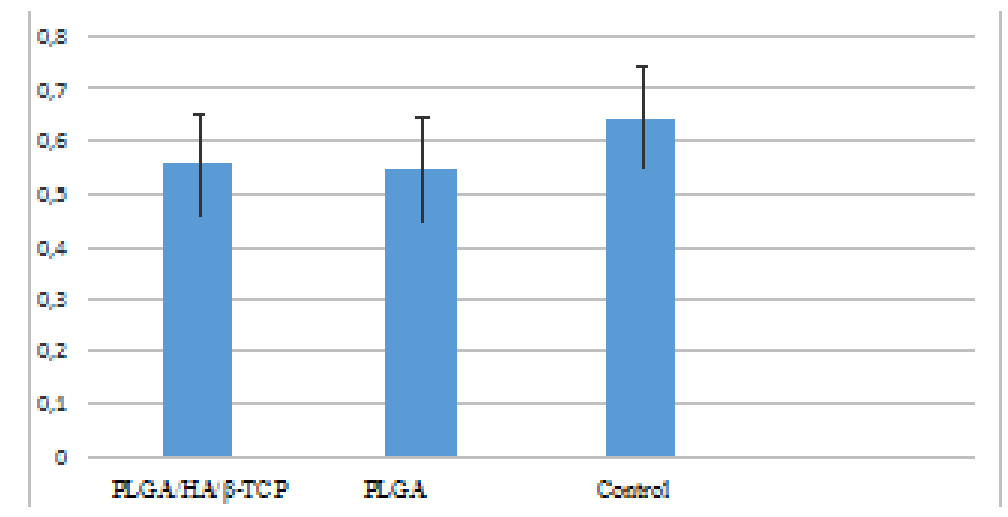

\section{Conclusion}

Our results can help understand the fundamental mechanisms of mesenchymal stem cells interactions with HA/ $\beta$ TCP ceramic in composition of composite scaffolds which depend of HA/ $\beta$-TCP structure, to enhance cell function and bone regeneration, and translate it to clinical implementation.

\section{Acknowledgements}

The investigation was funded by the Ministry of Science and Higher Education of the Russian Federation, agreement № 14.575.21.0164, ID number RFMEFI57517X.

\section{References}

[1] M. S. Lopes, A. L. Jardini, R. M. Filho, "Poly (Lactic Acid) Production for Tissue Engineering Applications," Procedia Eng., vol. 42, pp. 1402-1413, 2012.

[2] Y. A. Nashchekina, N. M. Yudintceva, P. O. Nikonov, E. A. Ivanova, L. V. Smagina, I. V. Voronkina, "Effect of Concentration of Collagen Gel on Functional Activity of Bone Marrow Mesenchymal Stromal Cell," Bull Exp Biol and Med, vol. 163, no. 1, pp. 123-128, 2017.

[3] Y. A. Nashchekina, T. V.Veselova, P. O. Nikonov, M. I. Blinova, "The study of the effect of polylactide scaffold and bone marrow stromal cells on reparative bone formation of the defect in the rabbit's mandible," Hum Gene Ther, vol. 26, no. 10, A-84, pp.195, 2015. 